\title{
Hemoptysis caused by erosion of thoracic aortic aneurysm
}

\author{
Dongmei Sun MSc MD, Sanjay Mehta MD FRCPC
}

Previously published at www.cmaj.ca

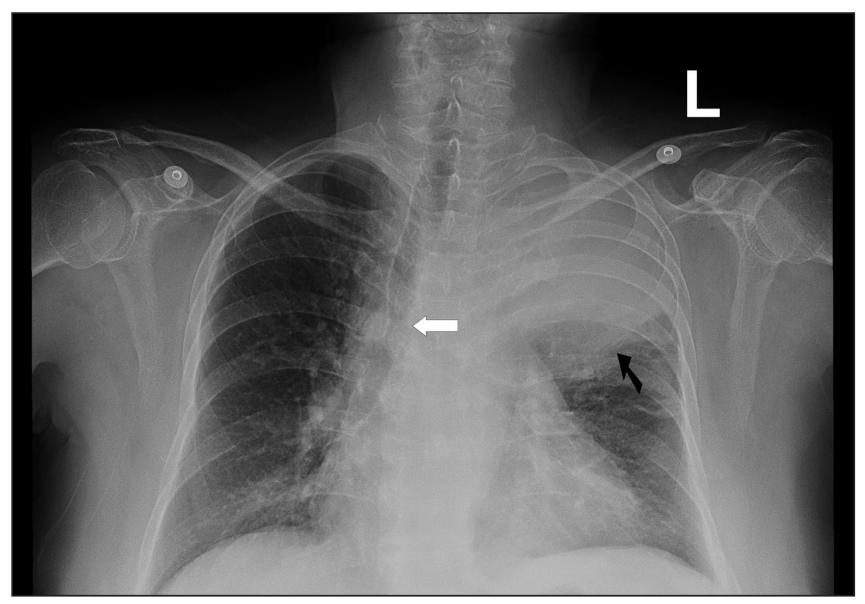

Figure 1: Chest radiograph of a 68-year-old woman showing opacification in the left upper lobe (black arrow) with tracheal displacement to the right (white arrow). 68-year-old woman presented with a five-month history of dry cough and intermittent fever. During the previous month, she had intermittent retrosternal chest pain radiating to her back. Two days before presentation, she had coughed up about $5 \mathrm{~mL}$ of blood on two occasions. She had type 2 diabetes and had never smoked. There was no history of trauma.

On examination, the patient looked well, and her blood pressure was equal bilaterally. She had a fever (temperature $39^{\circ} \mathrm{C}$ ), and oxygen saturation was $96 \%$ on room air. Her blood leukocyte level was elevated, at 15.6 (normal 4.0-10.0) $\times 10^{9} / \mathrm{L}$, and blood cultures gave negative results. A chest radiograph showed opacification in the left upper lobe with tracheal displacement to the right, which suggested a mass lesion (Figure 1). The presumptive diagnosis was a pulmonary tumour complicated by obstructive pneumonia. Bronchoscopic examination did not show any evidence of infection. A computed tomography scan of the chest showed a thoracic aortic aneurysm and a heterogeneous mass in the left upper lobe consistent with a large hematoma (Figure 2). Urgent endovascular repair was successful.

From the Southwest Ontario Pulmonary Hypertension Clinic, Division of Respirology, Departments of Medicine and Physiology/Pharmacology, London Health Sciences Centre; and the Program in Critical Illness Research, Lawson Health Research Institute, University of Western Ontario, London, Ont.

CMAJ 2010. DOI:10.1503/cmaj.090447

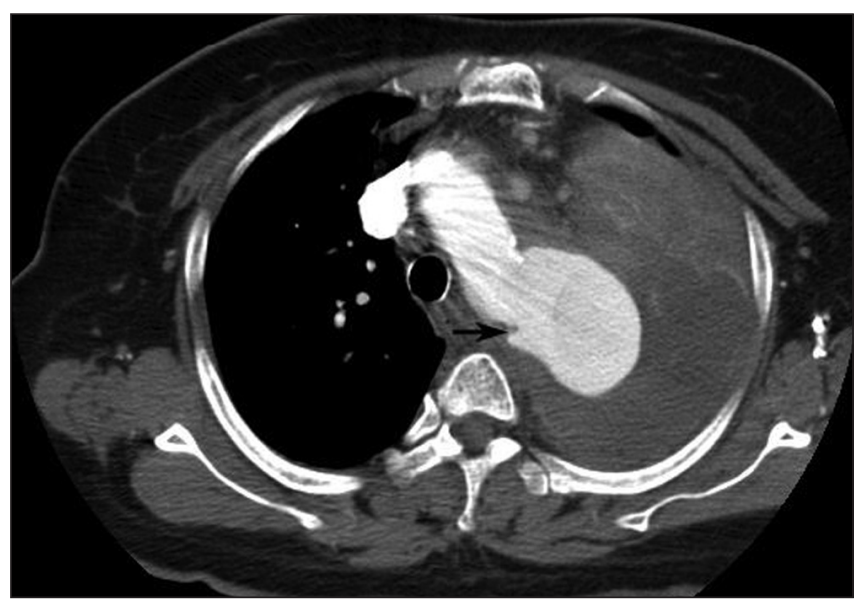

Figure 2: Computed tomography scan of the chest showing a thoracic aortic aneurysm $(4 \times 6 \mathrm{~cm}$, arrow) with a heterogeneous mass in the left upper lobe consistent with a large hematoma.

A dissecting aortic aneurysm usually presents acutely with dramatic symptoms, including severe chest pain. However, symptoms of a subacute or chronic thoracic aortic aneurysm can be nonspecific and caused by compression of adjacent structures. Hemoptysis has been reported following erosion of the aneurysm into the lung parenchyma. ${ }^{1}$ In this patient, fever was thought to be due to the inflammatory response to the hematoma. Common findings on a chest radiograph include a widened mediastinum and an enlarged aortic knob. Other reported atypical presentations of ruptured thoracic aortic aneurysm include painless hemorrhagic pleural effusion ${ }^{2}$ and tension pneumothorax from lung penetration. ${ }^{3}$

This article has been peer reviewed.

Competing interests: None declared.

\section{REFERENCES}

1. Fujii A, Swawmoto R, Misumi Y, et al. An autopsy case of massive hemoptysis due to a rupture of the thoracic aortic aneurysm into the left lung. Nihon Kokyuki Gakkai Zasshi 2009;47:57-60.

2. Little S, Johnson J, Moon BY, et al. Painless left hemorrhagic pleural effusion Chest 1999; 116:1478-80.

3. Kurosaki K, Fushimi Y, Hara S, et al. Sudden death caused by tension pneumothorax after rupture of a thoracic aortic aneurysm. Case report. Am J Forensic Med Pathol 2001;22:250-2. 